Bio-grafia Escritos sobre La Biología y su Enseñanza.

Edición Extra-Ordinaria. ISSN 2027-1034 P. p149-159

Memorias del VII Encuentro Nacional de Experiencias en la Enseñanza de la Biología y la Educación Ambiental y II Congreso Nacional de Investigación en la Enseñanza de la Biología

\title{
UNA PROPUESTA DE “ENSEÑANZA” DE LA EDUCACIÓN AMBIENTAL EN LA UPTC
}

\section{A PROPOSED “TEACHING” OF ENVERONMENTAL EDUCATION IN THE UPTC}

\section{Néstor Adolfo Pachón Barbosa ${ }^{1}$}

\section{Resumen}

El presente artículo presenta algunos apartes de la investigación realizada al quehacer docente propio, en la asignatura de educación ambiental, orientada a estudiantes de la Licenciatura en Ciencias Naturales y Educación Ambiental y de Ingeniería Ambiental, en el cual se propuso leer los aspectos relevantes de incorporar algunos cambios después del análisis metódico sobre las acciones pedagógicas emprendidas en la asignatura del año 2007 al 2013, donde se buscaba cambiar el tedio de las clases de salón, por otras que impulsaran la construcción conceptual y la generación de actitudes comunitarias más relevantes.

En esa etapa de cambio, se propusieron incorporar nuevos materiales de lectura, diversidad de temas de lo educativo ambiental, orientar proyectos de participación comunitaria así como contextualizar de mejor manera las prácticas de campo. Estas trasformaciones no solo desestabilizaron las concepciones estudiantiles, sino también las profesorales, al imprimirle unas concepciones prácticas y proyectivas, que suman retos a la función social del docente o futuro docente de la UPTC y su rol principal en la transformación de sus comunidades.

Este proyecto no pretende descubrir nada novedoso, sino contar una experiencia de transformación del pensamiento no solo estudiantil sino principalmente el del docente, con su rol social y sus implicaciones sociales.

Palabras clave: Educación Ambiental, pedagogía activa y social, y transformación comunitaria.

Keywords: environmental education, Social and active pedagogy, community transformation

\footnotetext{
${ }^{1}$ Magister en Educación. Docente de la Licenciatura en Ciencias Naturales y Educación Ambiental. Grupo de Investigación WAIRA Ambiente, Comunidad y Desarrollo. Universidad Pedagógica y Tecnológica de Colombia nestor.pachon@uptc.edu.co; nestorpachon72@yahoo.com
} 
Bio-grafia Escritos sobre La Biología y su Enseñanza.

Edición Extra-Ordinaria. ISSN 2027-1034 P. p149-159

Memorias del VII Encuentro Nacional de Experiencias en la Enseñanza de la Biología y la Educación Ambiental y II Congreso Nacional de Investigación en la Enseñanza de la Biología

\section{Abstract}

This article presents some excerpts from research to teaching work itself, the subject of environmental education, aimed at students of the Bachelor of Natural Science and Environmental Education and Environmental Engineering, in which he proposed to read the relevant aspects of incorporating some changes after the methodical analysis of pedagogical actions undertaken in the course of 2007 to 2013, which sought to change the tedium of the classroom setting, which reinforced by other conceptual construction and generation of relevant community attitudes.

At that stage of change, incorporate new materials proposed reading of the diversity of environmental education issues, community participation projects focus and context of best practices in the field. These transformations not only destabilized the conceptions students, but also the professorial, to impart a projective concepts and practices that add challenges to the social role of the teacher or future teacher in UPTC and its leading role in the transformation of their communities.

This project don't aims to discover anything new, but to have a transformational experience of thought not only the student but the teacher mainly with its social role and its social implications.

\section{Introducción}

En esta indagación no se pretende presentar el estado del arte de la Educación Ambiental (EA) en la Universidad Pedagógica y Tecnológica de Colombia (UPTC), ni proponer elementos metodológicos para hacer una mejor clase de EA en la Universidad, este estudio es una autocrítica de las maneras como estamos constituyendo a los sujetos sociales, nuestros profesionales, desde la óptica de una Universidad académica, teórica, modeladora y hegemónica del conocimiento, y su papel final como actores sociales del cambio de sus comunidades.

Desconocer la potencialidad de aprendizaje que nos han brindado y podrán brindar las comunidades de nuestro entorno cercano - y no tan cercano -, sería tratar de minimizar las acciones educativas exclusivamente al aula, reproduciendo los modelos tradicionales de aprendizaje escolarizado y no darle oportunidad a las múltiples facetas y matices que tiene aprender en contexto, con alto nivel de entropía y caos, que permite renovar pensamientos, consolidar convergencias y divergencias, reconocer diversidad y en últimas, docentes más pertinentes.

El trabajo presenta algunos apartes del análisis realizado al quehacer docente, la posibilidad de incorporar nuevas metodologías de trabajo e indagar con los estudiantes el impacto de estas acciones en su proceso de formación, no solo 
Bio-grafia Escritos sobre La Biología y su Enseñanza.

Edición Extra-Ordinaria. ISSN 2027-1034 P. p149-159

Memorias del VII Encuentro Nacional de Experiencias en la Enseñanza de la Biología y la Educación Ambiental y 11 Congreso Nacional de Investigación en la

$$
\text { Enseñanza de la Biología }
$$

conceptual, sino actitudinal, axiológico y proyectivo en la vida de los estudiantes de la UPTC, y en su rol social.

En el contexto anterior, se planta como pregunta central u orientadora: ¿Cuáles han sido los aportes de incorporar diversos procesos pedagógicos en la "enseñanza" de la Educación Ambiental (EA) en la UPTC?

\section{Metodología}

Para desarrollar esta indagación, se ha tomado el Paradigma de la Investigación Acción, entendida esta como "un estudio de una situación social con el fin de mejorar la calidad de la acción dentro de la misma" (Elliot, 1993; en Latorre, 2007. P24). Está en el campo educativo, se asume como una reflexión sobre las acciones humanas y las situaciones sociales vividas por el profesorado que tiene como objetivo ampliar la comprensión (diagnóstico) de los docentes y directivas de sus problemas prácticos y cotidianos. Las acciones resultantes de ésta, van encaminadas a modificar, mejorar o fortalecer la situación, dependiendo de la condición de los hallazgos y una vez que se logre una comprensión más profunda de los problemas. (Latorre, 2007. p24)

Para Kemmis (1984) la investigación - acción en el campo social y docente se constituye además de una ciencia práctica y moral, también como una ciencia crítica:

[...] una forma de indagación autoreflexiva realizada por quienes participan (profesorado, alumnado o dirección por ejemplo) en las situaciones sociales (incluyendo las educativas) para mejorar la racionalidad y la justicia de: a) sus propias prácticas sociales o educativas; b) su comprensión sobre las mismas; y c) las situaciones e instituciones en que estas prácticas se realizan (aulas o escuelas, por ejemplo).(Kemmis, 1984; en Latorre, 2007. p24)

Como podemos observar los teóricos han propuesto el proceso de Investigación - Acción, como una posibilidad no solo de identificar las situaciones sociales o educativas que están generando conflictos, sino que propone que todos los agentes de la educación sean artífices de su propio cambio y gestores de nuevas dinámicas tanto educativas, como sociales, en el marco de una comprensión crítica y reflexiva de estas realidades.

Para el propósito de este trabajo se utilizó una visión de la Investigación - Acción basado en el Triángulo de Lewin (1946), que comprende la necesidad de la Investigación, la Acción y la Formación, como tres elementos esenciales para el desarrollo profesional.

El modelo de Investigación - Acción utilizado para esta indagación, es el de espiral cíclico, que involucra los dos ejes: estratégico (acción y reflexión) y el 
Bio-grafía Escritos sobre la Biología y su Enseñanza.

Edición Extra-Ordinaria. ISSN 2027-1034 P. p149-159

Memorias del VII Encuentro Nacional de Experiencias en la Enseñanza de la Biología y la Educación Ambiental y 11 Congreso Nacional de Investigación en la

\section{Enseñanza de la Biología}

organizativo (planificación y observación), que permita en un triple ciclo no solo determinar las situaciones educativas, sino dimensionar las diferentes posibilidades de acción individual y colectiva, para el mejoramiento permanente de la Acción Educativa Ambiental. (Kemmis, 1989)

Figura 1. Momentos y Ciclos de la Investigación - Acción de Kemmis (1989)

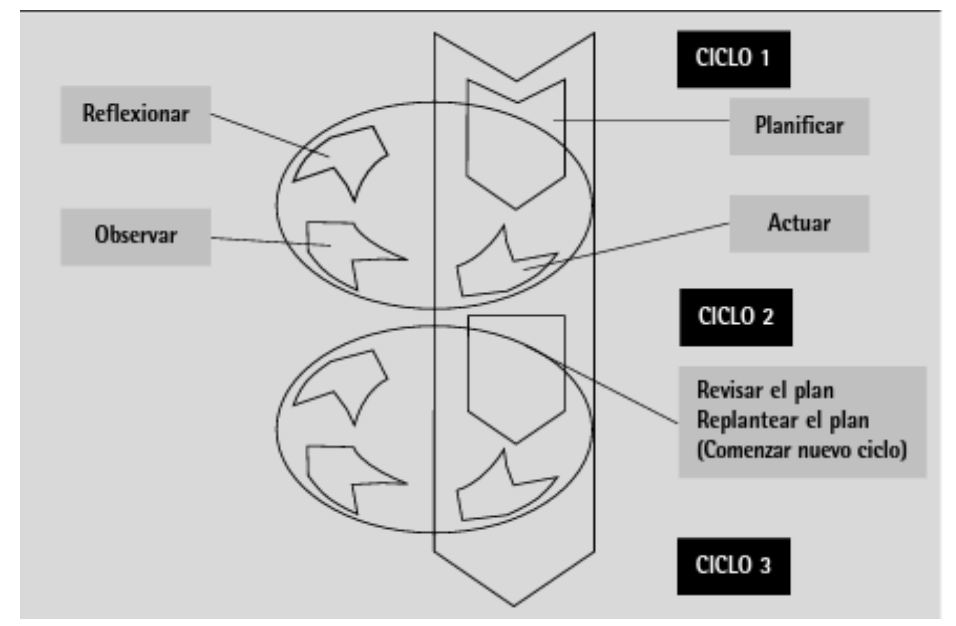

Fuente: Latorre, 2007

1.1. Instrumentos y técnicas. para la síntesis y análisis de la información fueron: Técnicas (Lectura Documental, Trabajo Colaborativo, Encuesta EA y Entrevistas SE). Instrumentos (Matrices de Análisis, Diagramas de Venn, Paralelos de Jerarquización y Cmap Tool)

1.2. Para este trabajo, el grupo objetivo fueron 5 grupos de educación ambiental de la Licenciatura en Ciencias Naturales y Educación Ambiental y 1 de Ingeniería Ambiental de la UPTC, como referente de observación de la acción educativa docente. Los estudiantes, fueron descritos como E1 al E125, por no tener aun permiso para publicar sus nombres.

1.3. Para le análisis de información, se realizó una triangulación (Figura 2) entre actores e instrumentos, para determinar fidelidad de la información y pertinencia para la investigación. Para consolidar el ciclo dos y tres, se trabajó con algunos análisis fenomenológicos sobre el trabajo con comunidad y de campo, basados entre lo que se escribe, se dice y se hace. (Figura 3). 
Bio-grafía Escritos sobre la Biología y su Enseñanza.

Edición Extra-Ordinaria. ISSN 2027-1034 P. p 149-159

Memorias del VII Encuentro Nacional de Experiencias en la Enseñanza de la Biología y la Educación Ambiental y II Congreso Nacional de Investigación en la Enseñanza de la Biología

Figura 2. Sistema de Análisis por Triangulación. secundario.

Figura 3. Sistema de Análisis

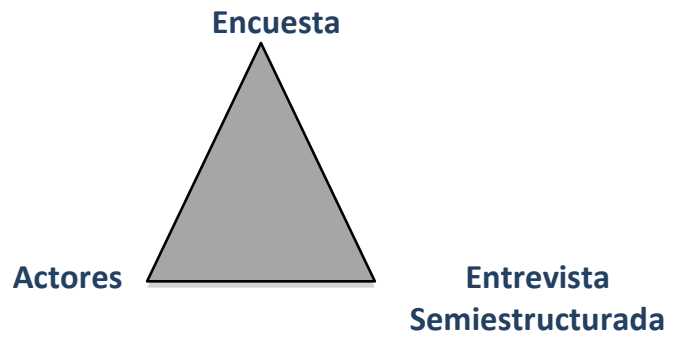

Fuente: Pachón-Barbosa, 2011.

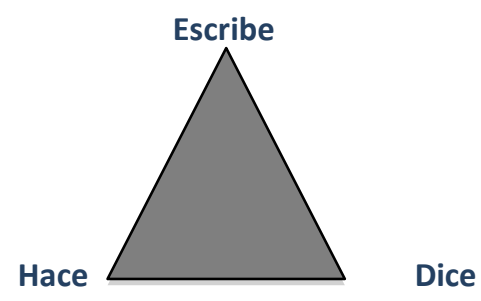

Fuente: Pachón-Barbosa, 2011.

\section{Análisis y discusión de Resultados.}

\section{Teorización y Praxis.}

En este primer escenario, se pudo observar en las encuestas y entrevistas informales que los estudiantes ven las clases magistrales como un esquema conocido, pero a la vez muy convencional y "aburrido", ya que los profesores solo se preocupan por "recitar" un concepto o contenido y la mayoría de veces no se preocupan por lo que piensa el estudiante o si existe comprensión. Como lo manifiesta el Estudiante 3 (E3) "el profe se preocupa por llenar tablero o dictarla clase, pero no está preocupado por los avances o por el estudiante”. En esta primera etapa en los momentos de Observación - Reflexión, se estableció una debilidad en la diversificación de los modos y formas de allegar el conocimiento docente y el de los estudiantes, para consolidar marcos teóricos complejos.

En la etapa de planificación se propuso algunas acciones de conceptualización, como son la lectura de material nuevo, artículos científicos, estudios de caso, entre otros y poder llevarlos al campo de la acción concreta en la comunidad escolar, pero también en comunidad de base.

Los estudiantes presentaron un cambio de actitud, ya que se mostraban más activos y con deseos de seguir abordando las lecturas y materiales, pero fueron dejando de lado la línea de trabajo, en la medida que los semestres iban avanzando temática y temporalmente, perdiéndose el influjo de las nuevas propuestas de trabajo y lectura. En el análisis de las encuestas y entrevistas, se pudo leer la emotividad de un nuevo método, pero la perdida de interés por la poca dedicación a sus materiales. En la Entrevista al E7 se percibe este cambio "En las lecturas nos pareció muy divertidas y profundas, pero luego no había tiempo o no me daba ganas de leerlas, ya que eran muchas y tengo otras asignaturas en el semestre". La evidente perdida de interés puede estar dada por 
Bio-grafia Escritos sobre La Biología y su Enseñanza.

Edición Extra-Ordinaria. ISSN 2027-1034 P. p149-159

Memorias del VII Encuentro Nacional de Experiencias en la Enseñanza de la Biologia y la Educación Ambiental y II Congreso Nacional de Investigación en la

\section{Enseñanza de la Biología}

varios factores, uno el desinterés por el material, cosa que se leyó en varias entrevistas. El otro por la poca cultura de lectura del estudiante, causado por clases magistrales y con poca participación.

En este sentido, se realizaron algunas encuestas y se encontraron tendencias sobre el papel del maestro (enseñar) y el del estudiante (aprender), la fragilidad de las relaciones entre estos dos actores y el papel protagónico que deberían tener los estudiantes en su construcción conceptual. A este respecto Ochoa (2013) asegura "la interacción entre sujetos, como constituyente de la relación pedagógica, se materializa en un proceso de enseñanza-aprendizaje. En este proceso, los sujetos se enfrentan a discursos y prácticas del saber y del quehacer social pero la relación entre ellos no es directa sino que es una relación siempre mediada, vehiculizada" (pág. 3) Es así como los estudiantes, todavía sienten que la "enseñanza" depende de un intermediario que es el docente y no consideran o no piensan que ellos pueden ser artífices del aprendizaje.

A partir de este análisis y de algunas pistas encontradas en las prácticas de campo de la asignatura, se entró en la segunda fase y se comenzó a ver la posibilidad de articular lo aprendido con las acciones del contexto social donde está inserta la Universidad o sus lugares de residencia habitual. Es así como en la fase de planeación, se incorporaron trabajos con comunidad, donde se respondiera a las problemáticas ambientales del contexto, desarrollando acciones y actividades de EA en estos sectores.

La transformación de los ejercicios de la asignatura, inicialmente generó algo de incertidumbre y debilidades en los estudiantes, por el acoplamiento a la actividad externa al aula; pero no fue distinto para el docente, que también sufrió algo de incertidumbre, temor de una nueva dinámica grupal, mayor trabajo de seguimiento y mayor preparación en el tema. Como menciona Torres (1997) sobre esta relación entre los factores que componen el ambiente y por ende la educación ambiental,

\footnotetext{
"De acuerdo con el carácter sistémico del ambiente, la educación ambiental debe ser considerada como el proceso que le permite al individuo comprender las relaciones de interdependencia con su entorno, con base en el conocimiento reflexivo y crítico de su realidad biofísica, social, política, económica y cultural, para que, a partir de la apropiación de la realidad concreta, se puedan generar en él y en su comunidad actitudes de valoración y respeto por el ambiente". (pág. 35)
}

En este sentido, reconstruir el conocimiento de éstas interrelaciones posibilita al estudiante y al profesor complementar su conocimiento, y permite así mismo dinamizar las acciones efectivas de la Universidad en su contexto en el tema educativo ambiental. 
Bio-grafía Escritos sobre la Biología y su Enseñanza.

Edición Extra-Ordinaria. ISSN 2027-1034 P. p149 - 159

Memorias del VII Encuentro Nacional de Experiencias en la Enseñanza de la Biología y la Educación Ambiental y II Congreso Nacional de Investigación en la

\section{Enseñanza de la Biología}

En los primeros tres grupos de la asignatura, se presentaron muchas dificultades para coordinar las acciones de los estudiantes, con los intereses de la comunidad, para lo cual se redireccionaron algunas acciones, como fueron apoyos conceptuales para el trabajo comunitario, herramientas de participación comunitaria y para la lectura de contexto, entre otras. Eso dejó evidenciar que el docente también debe volcar sobre sus estudiantes no solo todo su conocimiento, sino su experticia en el tema y en el manejo de comunidad, con el fin de conformar un bloque teórico - práctico, que permitiera al grupo de clase tener mayores elementos de trabajo con la comunidad.

Algunas de las observaciones de los docentes sobre este campo dejan entrever lo que antes se analizaba, como menciona E25 "al principal dificultad de trabajar con la comunidad fue el acercamiento inicial, ya que las comunidades son muy prevenidas y no muestran mucha confianza, lo que nos dejó sin piso para iniciar el trabajo y por eso algunos habitantes del barrio no volvieron a las actividades o no quisieron continuar los talleres". La primera expresión demuestra el escaso trabajo y preparación que les presentamos a los estudiantes para trabajar con comunidades diferentes a las escolares, así como la debilidad de nosotros de brindar alternativas pedagógicas para trabajo con comunidades no escolares.

En la tercera fase de trabajo, la etapa más dura para mí como docente, fue la reflexión de cómo superar algunos de estos obstáculos como docente y como estimular el trabajo y la conceptualización en los estudiantes, teniendo en cuenta las diferentes variables de la comunidad. Quizá la herramienta más útil fue seguir abordado los grupos desde las concepciones pedagógicas activas, pero adentrándonos aún más en las pedagogías sociales, para poder encontrar elementos de trabajo con las diferentes comunidades.

En este sentido, surgen inquietudes dentro de los estudiantes y del mismo profesor, de ¿cómo realizar esos procesos de inmersión en la comunidad?, ¿cuál es la función política de nosotros como educadores ambientales? y sobre todo, ¿esa es una función de la pedagogía y de la educación?

Así surge el último análisis y planificación de este proceso inacabado, y es la discusión conceptual y metodológica del papel de la educación ambiental y los modelos pedagógicos del maestro y sus estudiantes, puestos a disposición en el devenir de las comunidades. Y cuál es el papel del maestro, sino repensar ese diálogo entre la función educativa y sus modelaciones históricas, las acciones educativas y sus implicaciones en mantener las hegemonías de la sociedad y por ende los modelos de desarrollismo económico, que impactan fuertemente lo ambiental en las comunidades.

En coherencia con esta afirmación, Caballo y Gradaílle (2008) manifiesta "apostamos por una educación que no circunscriba sus iniciativas únicamente a 
Bio-grafía Escritos sobre la Biología y su Enseñanza.

Edición Extra-Ordinaria. ISSN 2027-1034 P. p149-159

Memorias del VII Encuentro Nacional de Experiencias en la Enseñanza de la Biología y la Educación Ambiental y II Congreso Nacional de Investigación en la

\section{Enseñanza de la Biología}

los espacios y contextos reglados por el diseño del currículum o los aprendizajes escolares, ampliando los horizontes de actuación y remitiéndonos a una concepción más integral, plural y compleja; esto es, desarrollando una acción socioeducativa que requiera de una reflexión-acción-intervención de naturaleza multidimensional e interdisciplinar, capaz de impulsar la apertura de los centros escolares a la sociedad, de promover y dinamizar una "sociedad que eduque y de una educación que socialice” (Ortega: 1999, 19)”. (pág. 46)

Es así como el grupo de dicentes y docente, se propone permitir enseñar, pero sobre todo aprender de la comunidad, como una pieza clave en el aprendizaje colaborativo y socioconstructivo, que catapulte las acciones de socialización y consolidación del conocimiento, pero sobre todo de resolución de problemas del contexto social, que antes parecían insuperables o con grandes dificultades de ser abordadas, comprendidas y resueltas. Este proceso mejoró las dinámicas de aprendizaje de estudiantes, como manifiesta E7 "La comunidad nos dio una lección de humildad y de dedicación que nosotros no teníamos como grupo, nos enseñó a trabajar en equipo, permitió encontrar soluciones a los problemas de residuos que había en el barrio, también aportaron muchos en tiempo y disposición, así uno se siente contento y seguro que los problemas ambientales del sector se van a mejorar."

En este contexto se puede decir que las teorías construidas en clase no son suficientes, si no están ligadas a experiencias de aprendizaje en el contexto, en la realidad, en lo práctico, que permitan ver consolidadas las teorías en hechos prácticos. E8 afirma, "la realidad en la mayoría de casos supera las teorías, ya que puede uno ver los verdaderos cambios en la sociedad, dimensionar las problemáticas e impulsar alternativas de solución como mencionó el profesor en clase". Así correlacionar las teorías, impulsar acciones y coparticipar con las comunidades, hace más fuerte y eficiente el proceso de "enseñanza aprendizaje".

\section{Fundamento Epistemológico.}

Las concepciones pertenecen a cuerpos epistemológicos de los saberes particulares y están ligados a procesos políticos, estratégicos e históricos, que le brindan una connotación particular y propiedades únicas. En el campo de la EA, estos elementos epistemológicos hacen que ella tenga unas concepciones muy particulares basadas en la visión sistémica del ambiente, la sostenibilidad (ambiental) en términos de calidad de vida, la interdisciplinariedad o el diálogo de saberes como fundamento para la comprensión del mundo, y la participación como agente transformador y de cambios en el tema ambiental. (Pachón, 2012)

Un aspecto importante en esta búsqueda epistemológica de la educación ambiental para ser construida y socializada en el contexto universitario, fue poder 
Bio-grafia Escritos sobre La Biología y su Enseñanza.

Edición Extra-Ordinaria. ISSN 2027-1034 P. p149-159

Memorias del VII Encuentro Nacional de Experiencias en la Enseñanza de la Biología y la Educación Ambiental y 11 Congreso Nacional de Investigación en la

\section{Enseñanza de la Biología}

ubicar un campo integral y reflexivo, que permita comprender las dinámicas ambientales, para entender los valores que subyacen al pensamiento ambiental y que son fundamentales para comprender las crisis de nuestro entorno natural, social y cultural como lo afirma Moreno-Yus (2007) en su pedagogía de la sostenibilidad:

Se hace, por ello, indispensable promover un pensamiento capaz de comprender la crisis de tipo económico, humano, social, medioambiental, educativa,... en la que nos sitúa, así como el papel que la educación ha de desempeñar en este contexto de insostenibilidad.

Reconocer estas interacciones sin haber construido un camino comunitario y social, sería casi imposible, ya que es necesario comprender las interacciones sociales, económicas, políticas y culturales que subyacen en las comunidades y que han generado las problemáticas ambientales, pero que también pueden potencializar las alternativas de solución a dichos problemas.

Posibilitar estas acciones de teorización en el aula, proyectos con la comunidad y visualización en las prácticas de campo con los estudiantes, permitió un mejoramiento en las relaciones docente - estudiante, permeo la estructura tradicionalista de lo escolar, amplió la visión del estudiante como futuro profesional, e impulso una serie de trabajos de grado y pasantías en esta asignatura, que puede garantizar algo efectivo en la práctica.

Recontextualizar la función docente y el papel casi liberador de transformar los espacios educativos, en campos de intervención cualificada con la comunidad, permite engendrar la semilla trasformadora de la sociedad, no solo en lo ambiental, sino en general en las necesidades que tiene la comunidad, desde las imperiosas materiales, como aquellas de lo espiritual y metafísico.

Para finalizar consolidar unas actividades teóricas, los proyectos comunitarios y las prácticas de campo, permite a los estudiantes interactuar en diferentes contextos para su construcción conceptual, pero sobre todo permite al maestro explorar otros caminos, otras concepciones de trabajo, otras dinámicas de clase, otros campos axiológicos, pero sobre todo permite impulsar al docente a la constante búsqueda de nuevas maneras de hacer de la educación un camino agradable y diverso. 
Bio-grafia Escritos sobre La Biología y su Enseñanza.

Edición Extra-Ordinaria. ISSN 2027-1034 P. p149-159

Memorias del VII Encuentro Nacional de Experiencias en la Enseñanza de la Biología y la Educación Ambiental y 11 Congreso Nacional de Investigación en la Enseñanza de la Biología

\section{Conclusiones.}

El principal aporte de la propuesta de incorporar procesos de conceptualización nueva, incorporando proyectos de extensión comunitaria y prácticas de campo contextualizadas, han permitido transformar el significado de la acción educativa pertinente y con impacto en las comunidades.

La incorporación de actividades enfocadas en los modelos pedagógicos activo y social, permitieron considerar las actividades educativas, no como una estrategia para consolidar conocimiento conceptual, sino poner de manifiesto el papel principal de la educación ambiental y sus destinatarios, como actores de transformación social en sus comunidades.

Las políticas nacionales e institucionales en el tema de la EA, han incluido estos componentes desde su promulgación en el año 96 en Colombia, pero no han sido implementadas de manera definitiva y contundente en los espacios de formación Universitaria.

El proceso sirvió a los estudiantes como sistema de evaluación del programa y de construcción de una mirada crítica y reflexiva de la actividad tanto del docente, como de ellos mismos como estudiantes. El paso de la teoría a la práctica, fue un poco complejo en la medida que generó incertidumbres, conflictos y controversias que se fueron limando en la medida que fueron discutidos en clase.

Por ultimo este trabajo, sirvió de referente al maestro para estar realizando transformaciones permanentes en sus actividades de clase, imprimiéndole una dinámica nueva a las acciones educativas de sus clases, e impulsando la consolidación de sujetos sociales más activos e interesados en las problemáticas de su contexto.

\section{Bibliografía}

AMAYA, G. (2013) La Pedagogía Activa. Procesos del conocimiento e implicaciones en las tareas del aula. Encontrado en http://www.pedagogica.edu.co/storage/ps/articulos/pedysab04 07arti.pdf Consultado el 31 de Julio de 2013

CABALLO, B. y GRADAÍlLE, R. (2008). La educación social como práctica mediadora en las relaciones escuela-comunidad local (Pág. 45-55). En Revista Interuniversitaria de Pedagogía Social (issn-1139-1723) no 15 - tercera época. Santiago de Compostela, España.

CONSEJO NACIONAL AMBIENTAL. (2002) Política Nacional de Educación Ambiental. Fotolito America. Bogotá, Colombia. 
Bio-grafia Escritos sobre La Biología y su Enseñanza.

Edición Extra-Ordinaria. ISSN 2027-1034 P. p149-159

Memorias del VII Encuentro Nacional de Experiencias en la Enseñanza de la Biología y la Educación Ambiental y 11 Congreso Nacional de Investigación en la

\section{Enseñanza de la Biología}

LATORRE, A. (2007) La Investigación - Acción: conocer y cambiar la práctica educativa. Editorial GRAO. Barcelona, España.

PACHÓN-BARBOSA, N. (2012). Educación Ambiental (EA) en la Institución Educativa Integrada de Combita, Boyacá - Colombia: diagnóstico preliminar. En Memorias del $5^{\circ}$ Congreso Internacional por el Desarrollo Sostenible y el Medio Ambiente. Universidad de Manizales. Manizales, Colombia.

MORA-PENAGOS, W. (2009) Educación ambiental y educación para el desarrollo sostenible ante la crisis planetaria: demandas a los procesos formativos del profesorado. En Revista Tecné, Episteme y Didaxis (TED) No. 26, 2009. Universidad Pedagógica Nacional. Bogotá, Colombia.

MORENO-YUS, Ma A. (2007) Pedagogía para la sostenibilidad basada en la recuperación de tres vínculos: vital, humano y natural. En Educatio Siglo XXI, No 25 • 2007, pp. 167186. Universidad de Murcia. Murcia, España. 